\section{Alexandra Popa}

Holistic Dental \& Medical Institute of Bucharest - ROPOSTURO,

Bucharest, Romania

e-mail: alesandra17popa@gmail.com

\section{Dental Management of the Pregnant Patient}

Editor: Christos A. Skouteris

Publisher:Wiley-Blackwell, Hoboken, NJ, USA

Language: English

ISBN: 978-1-119-28656-1

Edition: $1 / \mathrm{e}$

Publish Year: 2018

Pages: 192, illustrated

Price: $€ 88.90$

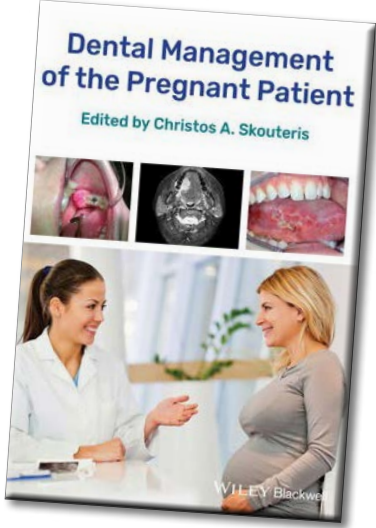

The physiological changes that occur during pregnancy must be known to the dental practitioner for him to know how to plan and treat pregnant patients. Dr. Christos A. Skouteris (University of Michigan School of Medicine in Ann Arbor, USA) and the 6 contributors, draft their first book, Dental Management of the

Pregnant Patient, on how to approach such patients. The book is divided into 9 chapters, plus a series of appendices, a Self-Assessment Quiz and an Index. The presentation of the ethical issues in the treatment of the pregnant patient is followed by the physiologic changes and their implications in the dental management of pregnant patients: cardiovascular, respiratory, hematologic, gastrointestinal, genitourinary, endocrine, immunologic, dermatologic, musculoskeletal, psychologic and behavioral changes. There follows a presentation of the general principles for the comprehensive treatment of pregnant patients, including prenatal counseling and prevention. A special place is occupied by the dental, oral, and maxillofacial diseases and conditions and their treatment. The book includes a description of each treatment of dental diseases, odontogenic oral and maxillofacial infections, benign diseases and conditions management of oral and maxillofacial malignancy and management of oral and maxillofacial trauma. The book tackles known postnatal considerations on medical contraindications, infant oral health and procedures and medications during breastfeeding, Basic Life Support (BLS) and Advanced Cardiac Life Support (ACLS) in pregnancy and obstetric gynecologic emergencies. The book provides information on emergency medical and gynecological issues in pregnant patients, and is an indispensable guide for any practitioner who is treating a pregnant woman.

d) http://www.stomaeduj.com 10.25241/stomaeduj.2020.7(3).bookreview.5

\section{Basic Guide to \\ Dental Sedation Nursing}

Author: Nicola Rogers

Language: English

ISBN: $978-1-119-52577-6$

Edition: $2 / \mathrm{e}$

Publish Year: 2020

Pages: 200, illustrated

Price: $€ 45.20$
Publisher:Wiley-Blackwell, Hoboken, NJ, USA

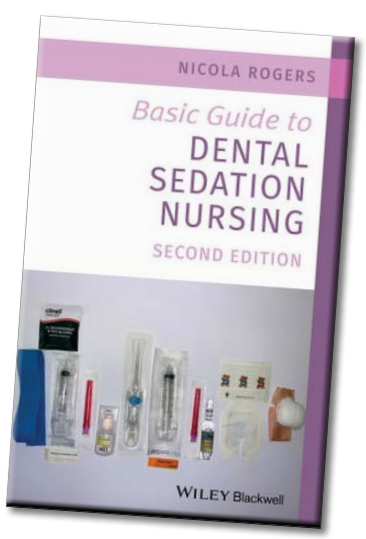

Nowadays, more and more patients request dental treatments under sedation. In the book entitled Basic Guide to Dental Sedation Nursing, 2nd Edition, Nicola Rogers, dental nurse tutor for pre and post-registration qualification training at Bristol Dental Hospital in Bristol, UK presents the basic information that any dental nurse needs to know in order to be able to help the treatments performed under dental sedation. The book is divided into seven chapters, accompanied by an index. It presents the most important aspects of sedation one by one, such as the definition of conscious sedation, medico-legal aspects of dental sedation, the role of the dental nurse and equipment, patient selection, types of sedation, medical emergencies and finally essential anatomy regarding the blood, the heart, and the respiratory system. The book is accompanied by clear images and diagrams so that the reader could understand the information presented much faster and easier. It provides good support for any dental nurse who wants to participate in treatments performed under sedation and is also a very good manual for sedation nurses undertaking any of the current National Examining Board of Dental Nurses (NEBDN) sedation qualifications, including the NEBDN Diploma in Dental Nursing, City and Guilds or CACHE Dental Nursing qualifications, as well as for dental hygienists and therapists.

The Books Review is drafted in the reviewer's sole wording and illustrates his opinions. 